Mots. Les langages du politique

$70 \mid 2002$

La politique en chansons

\title{
Propositions pour une méthode d'analyse du discours télévisuel
}

Some propositions for a method of Discourse analysis of television discourse

Propuestas para un método de análisis del discurso televisual

\section{Marlène Coulomb-Gully}

\section{OpenEdition}

Journals

Édition électronique

URL : https://journals.openedition.org/mots/9683

DOI : $10.4000 /$ mots.9683

ISSN : 1960-6001

Éditeur

ENS Éditions

Édition imprimée

Date de publication : 1 novembre 2002

Pagination : 103-113

ISBN : 2-84788-016-X

ISSN : 0243-6450

\section{Référence électronique}

Marlène Coulomb-Gully, «Propositions pour une méthode d'analyse du discours télévisuel », Mots. Les langages du politique [En ligne], 70 | 2002, mis en ligne le 30 mars 2011, consulté le 22 avril 2022. URL : http://journals.openedition.org/mots/9683 ; DOI : https://doi.org/10.4000/mots.9683

\section{(c) ENS Éditions}


Marlène Coulomb-Gully ${ }^{\circ}$

\section{Propositions pour une méthode d'analyse du discours télévisuel}

Je me propose d'aborder les notions de méthodes et de corpus en les rapportant au champ qui est le mien, à savoir le discours d'information télévisuel ${ }^{1}$. L'étude du discours médiatique et plus particulièrement télévisuel est investie par des chercheurs venant d'horizons très différents (sociologie, science politique, linguistique, histoire, etc.) mais très souvent des sciences sociales; le type d'approche méthodologique et la définition du corpus les plus largement répandus dans ce type d'études sont en partie tributaires de la formation des chercheurs et des méthodes dominantes dans leur discipline d'origine. Venant moi-même de l'analyse littéraire, c'est donc un regard sur ces questions que je proposerai, probablement différent de celui de l'historien confronté à ces mêmes questions.

Je voudrais souligner aussi la jeunesse de ce secteur d'études. Si les collègues qui travaillent sur le discours écrit ont derrière eux une tradition de recherche plusieurs fois millénaire, ceux qui comme moi s'intéressent au discours audiovisuel s'appuient sur quelques dizaines d'années d'études tout au plus. Ceci précisé, je vais au cours de cet exposé aborder d'un point de vue nécessairement schématique, voire elliptique parfois, les questions de méthodes et de corpus qui se posent à nous.

- Université de Toulouse 2 - Le Mirail.

1. Cet article reprend l'exposé oral fait lors de la Journée d'étude de Mots consacrée à l'approche des corpus en AD (Montpellier novembre 2001). Je remercie D. Maingueneau de sa relecture attentive. 


\section{Théories critiques et discours télévisuel}

Ce préambule historique peut paraitre redoutable quand on a la prétention d'être bref; il n'en est pas moins incontournable, moins par souci d'érudition que parce qu'il va permettre, par le biais de ses approches critiques, de cerner les spécificités du discours télévisuel et finalement des méthodologies qui me paraissent le plus appropriées à son étude.

\section{Les années 70 et le structuralisme}

Les premiers travaux critiques sur l'image animée ont porté sur le cinéma (André Bazin étant une des références majeures dans ce domaine), mais je m'arrêterai un instant sur les années 60-70 et l'approche sémiotique dont Christian Metz a été un représentant fameux. Rappelons-nous l'article célèbre dans lequel, considérant son objet de façon totalement autonome, le critique lui appliquait des principes d'analyse strictement langagiers ${ }^{2}$. C'est la grande époque du structuralisme où le modèle linguistique est dominant, pour ne pas dire totalitaire. Le principe du structuralisme, c'est l'immanence; l'objet est étudié « en soi », indépendamment de ses conditions de production, de réception, etc.

Quel est l'intérêt de cette mise en perspective pour l'analyse du discours télévisuel?

$\mathrm{Au}$ regard de ce type d'approche, la télévision ne présente que peu d'intérêt. En effet, si ses qualités esthétiques font qu'un film peut être appréhendé comme une structure fermée, de même qu'un poème voire un roman, pour la télévision, l'essentiel n'est évidemment pas là. D'où sans doute le fait que durant cette période, la télévision n'est ou pas étudiée du tout, ou considérée comme un sous-genre par rapport au cinéma, sousgenre aux caractéristiques strictement négatives : répétitivité, stéréotypie, standardisation, etc. En fait, il va falloir attendre la fin des années 70 et le début des années 80 pour observer un changement d'attitude face à la télévision.

\section{Les années 80 et la pragmatique}

On observe à cette époque un changement de paradigme sur le plan de la théorie critique. En effet, on est de plus en plus conscient des limites de à 90 .

2. Ch. Metz, «Le cinéma : langue ou langage? », Communications $n^{\circ} 4,1964$, p. 52 
ce structuralisme radical que j'ai évoqué précédemment, et l'on redécouvre l'intérêt qu'il y a à rattacher un texte à son contexte, en amont du côté de la production comme en aval du côté de la réception. C'est la période où la linguistique qui constitue toujours une discipline motrice sur le plan théorique, découvre, avec Austin entre autres, la pragmatique.

De façon encore une fois très schématique, on peut dire que la force de la pragmatique, c'est de relier un texte à son contexte, de considérer que tout énoncé doit être relié au travail d'énonciation en dehors duquel il n'est pas compréhensible.

On voit ce que la télévision a à gagner à ce déplacement de perspective, la spécificité du média résidant en effet dans les conditions de production, de circulation et de réception des messages. Cette caractéristique, impossible à intégrer dans une approche de type structural, trouve dans les approches pragmatiques un cadre idéal. En même temps qu'elle affirme sa spécificité par rapport au cinéma, la télévision s'affirme comme objet d'étude à part entière ${ }^{3}$.

Ce que je viens de dire présuppose que certains genres de discours sont plus en phase avec une méthode d'analyse qu'avec d'autres; ou, formulé différemment, que certaines méthodes s'avèrent plus productives face à certains types de discours que face à d'autres. Le structuralisme était particulièrement opératoire face à une structure close comme un poème, la pragmatique est plus opératoire face à des structures ouvertes, comme le discours télévisuel.

\section{Démarche pragmatique et analyse du discours télévisuel}

$\mathrm{Au}$ début des années 80 donc, la recherche sur la télévision trouve un cadre d'étude approprié en même temps que celui-ci révèle la spécificité du média dont les conditions de production, de circulation et de réception du message sont fondamentales.

L'étude de la télévision requiert d'emblée une démarche d'obédience pragmatique, i.e. qui intègre le discours télévisuel dans ses co-textes et son contexte de communication, le contexte n'étant jamais simplement placé autour d'un énoncé, mais structurant l'énoncé.

3. Voir Hors cadre $n^{\circ}$ 7, 1989, « Théorie du cinéma et crise de la théorie ». 


\section{Le texte télévisuel dans son contexte de communication}

- En amont : les conditions de production

Le discours d'information télévisé qui m'intéresse en priorité ici, est indissociable, en amont, des conditions de production; je ne m'étendrai pas sur ce point, mais il est clair que les collectifs professionnels, les professionnels des médias et les équipes journalistiques - si tant est que l'on puisse globaliser l'approche de tous ces groupes -, constituent un premier élément à prendre en compte dans la structuration du discours d'information.

Par exemple, les informations diffusées sur TF1 ne doivent pas être en contradiction avec les intérêts du groupe Bouygues, comme on l'a vu dans la couverture de la guerre Iran/Irak ou dans l'absence de couverture de la contestation des usagers du périphérique lyonnais TEO, dans la construction duquel Bouygues-BTP était partie prenante ${ }^{4}$.

- En aval se pose la question de la réception et plus spécifiquement celle des usages de la télévision et de l'interprétation des messages :

- Du côté des usages, on ne comprendrait rien aux choix des sujets abordés aux informations de 20 heures, par exemple, si l'on faisait abstraction du fait que leur horaire de diffusion en fait un spectacle familial $^{5}$ : pas d'information qui choque, une organisation qui emprunte à la logique narrative, qui veut que l'on termine par un sourire, sur des informations « douces ».

C'est aussi cette logique des usages qui a condamné la diffusion de films pornographiques sur la Cinq (la Cinq première version, celle de Berlusconi) le mardi soir : le mardi soir, les enfants qui n'ont pas cours le mercredi, veillent... Et qui, pour des raisons opposées, a assuré le succès de ceux de M6 le dimanche soir : les enfants ayant classe le lundi, se couchent tôt la veille...

- Du côté de l'interprétation, le programme fonctionne comme proposition de sens, même si l'appartenance sociale du téléspectateur est fondamentale dans la réception et le décryptage du message. C'est ainsi

4. Il existe de très nombreux travaux, plus ou moins scientifiques, sur cet aspect. Ainsi du très bon numéro du Nouvel Observateur de juillet 1999, qui propose un panorama exhaustif des relations entre capitaines d'industrie, groupes de communication et médias. Voir aussi pour la seule chaîne TF1, TF1, Un pouvoir de P. Péan et Ch. Nick, Paris, Fayard, 1995. Du côté des journalistes, voir S. Halimi, Les nouveaux chiens de garde, Liber Raison d'agir, Paris, 1997.

5. Les anglo-saxons parlent à ce propos de «less objectionable program ». 
que l'émission Questions à domicile (présentée sur TF1 par Anne Sinclair et Pierre-Luc Séguillon puis Jean-Marie Colombani dans les années quatre-vingt) a été moins reçue comme une émission politique, telle que l'avaient conçue ses producteurs, que comme une émission "people » permettant d'entrer dans l'intimité des "grands »; d'où en retour un jeu de la part des personnalités politiques elles-mêmes avec le principe de l'émission, celles-ci refusant d'être filmées chez elles, mais sur leur lieu de travail, etc. ${ }^{6}$.

\section{Le texte télévisuel et son co-texte de communication}

Les informations télévisées, et le discours d'information en général, sont un moment de la grille télévisuelle et ses caractéristiques se sont façonnées par rapport aux autres genres télévisés autant que par rapport aux autres discours d'information (presse, mais surtout radio et actualités cinématographiques).

Le contrat à la base du discours d'information est fondé sur un impératif de crédibilité dont témoigne toute la scénographie du JT : l'obsession référentielle, le rôle du direct, la caution du terrain et des reporters, un discours enfin qui s'organise intégralement autour d'un imaginaire de la vérité (exhaustivité, neutralité, pluralité des points de vue, etc.).

Mais parallèlement à cet impératif de crédibilité à la base du contrat d'information, le discours d'information doit être produit selon les règles discursives propres au média télévisuel pour ne pas être contraires au principe de captation au fondement du discours TV dans son ensemble : d'où la présence d'un présentateur vedette et toute la mise en scène du contact (« les yeux dans les yeux »- axe Y-Y -, surenchère énonciative, etc.) qui fonde le JT, la mise en intrigue de l'information (l' " angle » avec un héros, la narration, " des stories, coco »), la dramaturgie du suspens, etc. ${ }^{7}$

Façonné par son contexte et son co-texte de communication, le discours télévisuel ne peut être appréhendé en dehors de ces éléments qui le structurent de part en part. On le voit, le contexte, ici pas plus qu'ailleurs, n'est jamais simplement placé autour d'un énoncé, mais il est premier dans le sens de l'énoncé : il n'y a de discours - médiatique/télévisuel que contextualisé.

6. Voir à ce propos l' article de B. Le Grignou et É. Neveu, «Émettre la réception, préméditation et réceptions de la politique télévisée », in Réseaux, Hors série, 1991, p. 65 à 94.

7. Sur les impératifs de crédibilité et de captation au principe du discours d'information, voir, de P. Charaudeau, Le discours d'information médiatique, INA-Nathan, 1997. 
Une fois posé le fait que le discours télévisuel, le discours d'information télévisuel est impensable en dehors de ces éléments co-textuels et contextuels qui le fondent et le structurent de part en part, quelles sont les conséquences méthodologiques et en terme de corpus que cela implique?

\section{Méthodes - Corpus}

\section{Analyse de discours/analyse de contenu}

On vient de le voir, les conditions de production du discours télévisuel, les conditions de circulation et de réception ne sont pas de simples circonstances annexes au discours, qui exerceraient sur lui des contraintes extérieures, mais elles sont structurantes, constitutives de ce discours, elles l'informent, au sens de «donner forme ». Cela veut dire que méthodologiquement, dans l'analyse que je fais du discours télévisuel, je dois m'efforcer de conjoindre en permanence ces différents plans de l'activité énonciative.

Cette posture qui consiste à considérer le travail d'énonciation (production/réception) comme constitutif de la production énonciative et à refuser la séparation énoncé/énonciation a été formalisée dans ce qu'on a coutume de nommer l'analyse de discours (AD), par opposition à l'analyse de contenu (AC).

Il se trouve que l'AC est très pratiquée en information-communication et en particulier dans l'analyse des discours télévisuels, notamment dans les milieux issus des sciences sociales. Et pour les raisons que j'ai dites plus haut, je crois que cette méthode est moins pertinente que l'AD, sauf peut-être dans le cas d'études longitudinales - diachroniques ${ }^{8}$.

On peut définir l'AC, en reprenant les termes de son initiateur, Bernard Berelson, comme « une technique de recherche pour la description objective, systématique et quantitative du contenu manifeste des communications, ayant pour but de les interpréter $»^{9}$. L'analyse de discours en revanche n'abordera pas séparément ce que l'AC appelle "le contenu manifeste des communications » d'une part et la situation de communica-

8. Sur ce postulat méthodologique, voir aussi G. Lochard, J.-Cl. Soulages, La communication télévisuelle, A. Colin, 1997; F. Jost, Introduction à l'analyse de la TV, Ellipses, 1999 .

9. Content analysis in communication research, GlencoeIII : Free Press, 1952, p. 18, repris par M. Grawitz, Méthode des sciences sociales, Paris, Dalloz, 1972. Voir aussi de Jean de Bonville, L'analyse de contenu des médias, de Bœck Université, 2000 . 
tion d'autre part, mais s'efforcera de les associer intimement. Dans l'AD, « toute activité énonciative est rapportée à un genre de discours : le lieu social dont il émerge, le canal par lequel il passe (oral, écrit, télévisé...), le type de diffusion qu'il implique, ne sont pas dissociables de la façon dont le texte s'organise $\gg{ }^{10}$.

L'entreprise est peut-être téméraire, mais je voudrais tenter, en m'aidant d'une étude sur cette question effectuée par P.Favre, de récapituler les principales oppositions qui me paraissent exister entre les deux approches et pour achever de montrer pourquoi l'AD me parait mieux adaptée à l'analyse du discours télévisuel que l'AC ${ }^{11}$.

\begin{tabular}{|l|l|}
\hline \multicolumn{1}{|c|}{ Analyse de contenu } & \multicolumn{1}{c|}{ Analyse de discours } \\
\hline née des sciences sociales & $\begin{array}{l}\text { née de la linguistique (d'une rupture } \\
\text { interne à la linguistique/Harris) }\end{array}$ \\
\hline $\begin{array}{l}\text { postule la transparence du discours par } \\
\text { rapport au réel }\end{array}$ & $\begin{array}{l}\text { postule l'opacité d'un discours auto- } \\
\text { nome par rapport au réel }\end{array}$ \\
\hline $\begin{array}{l}\text { les critères d'analyse sont exogènes au } \\
\text { discours étudié : caractérisation des } \\
\text { messages à partir de critères définis } a \\
\text { priori (méthode déductive) }\end{array}$ & $\begin{array}{l}\text { critères d'analyse endogènes, nés de la } \\
\text { lecture du texte analysé - méthode } \\
\text { inductive - (même si le savoir accumulé } \\
\text { permet aujourd'hui de définir certains } \\
\text { critères d'analyse a priori ) }\end{array}$ \\
\hline $\begin{array}{l}\text { suppose l'atomisation du discours : les } \\
\text { éléments du texte sont distribués dans } \\
\text { des catégories, on travaille sur des unités } \\
\text { isolées (mots...) même s'il existe de } \\
\text { plus en plus de logiciels qui prennent en } \\
\text { compte la structure textuelle }\end{array}$ & $\begin{array}{l}\text { lée; la structure est considérée comme } \\
\text { première dans le sens du texte }\end{array}$ \\
\hline $\begin{array}{l}\text { approche quantitative (comptage, } \\
\text { notions de fréquence, moyenne, écart- } \\
\text { type, etc.) }\end{array}$ & approche principalement qualitative \\
\hline $\begin{array}{l}\text { on postule la représentativité statistique } \\
\text { des textes }\end{array}$ & $\begin{array}{l}\text { on privilégie les conditions de produc- } \\
\text { tion, de circulation et de réception des } \\
\text { discours analysés }\end{array}$ \\
\hline
\end{tabular}

10. D. Maingueneau, Analyser les textes de communication, Dunod, 1998, p. 2.

11. P. Favre, « Analyse de contenu et analyse de discours - Sur quelques critères distinctifs ", in Études offertes au Professeur É. de Lagrange, Université de Clermont I, 1978. 


\begin{tabular}{|l|l|}
\hline $\begin{array}{l}\text { les analyses sont de type statistique et } \\
\text { thématique }\end{array}$ & $\begin{array}{l}\text { l'analyse privilégie l'activité énonciative } \\
\text { rapportée à un lieu et à un genre }\end{array}$ \\
\hline corpus : homogène et exhaustif & $\begin{array}{l}\text { homogénéité et exhaustivité non néces- } \\
\text { saires }\end{array}$ \\
\hline $\begin{array}{l}\text { reproductibilité de la méthode (parce } \\
\text { que les critères d'analyse sont exogènes } \\
\text { aux discours analysés) }\end{array}$ & $\begin{array}{l}\text { pas de reproductibilité systématique de } \\
\text { la méthode, chaque discours engendrant } \\
\text { les critères de son analyse, même si le } \\
\text { savoir accumulé permet une certaine } \\
\text { reproductibilité }\end{array}$ \\
\hline
\end{tabular}

Ces oppositions sont évidemment schématiques, d'autant plus que les deux paradigmes semblent de moins en moins étanches (l'AC tente de prendre en compte la question de la réception et de la production des discours qu'elle étudie, bien qu'elle le fasse de façon externe à l'analyse proprement dite, qui continue de se déployer de façon autonome) mais elles permettent, je crois, de mieux cerner l'intérêt et la pertinence de l'AD s'agissant de l'approche du discours télévisuel.

\section{La méthode et ses conséquences sur la définition et le choix du corpus}

On conçoit que pour l'AC, le corpus doive être homogène et exhaustif, ou alors fondé sur des principes d'échantillonnage satisfaisant à des conditions de représentativité, le principe étant que la partie est supposée refléter le tout.

L'AD pose différemment la question du corpus, l'accent est mis ailleurs. L'exhaustivité, pas plus que l'homogénéité ne sont pour elle un objectif; ce sont plutôt des moments privilégiés, des passages spécifiques d'un corpus qui l'intéressent, des lignes de fracture où s'exprime au mieux le lien entre énoncé et énonciation, le rapport entre émission, production et réception. Je dirais même que l'hétérogénéité, ou en tout cas la tension entre l'homogène et l'hétérogène, est ce qui fonde le corpus en $\mathrm{AD}$, l'objectif étant de mettre en évidence un système de différences - de " différances »? - dans les conditions de production, de circulation et de réception des discours.

Ainsi par exemple dans la célèbre étude de Véron, où celui-ci analyse le fonctionnement du dispositif d'énonciation propre aux informations télévisées ${ }^{12}$. Pour ce faire, il va choisir des moments charnières du dis-

12. É. Véron, « Il est là, je le vois, il me parle », Communications, 38, 1983, p. 98-120. 
cours audio-visuel où s'exprime le plus clairement la stratégie de captation du téléspectateur par le présentateur (il le regarde « les yeux dans les yeux » et guide son regard vers d'autres lieux), qui constitue en fait la spécificité du dispositif d'énonciation au JT.

Confrontée à cette même question (caractériser le dispositif d'énonciation propre au JT), l'AC aurait peut-être analysé tous les JT, minute par minute, ou seconde par seconde, selon un protocole où auraient été pris en compte tous les gestes, toutes les paroles, tous les regards des différents intervenants, etc.

Mais plus vraisemblablement, elle ne se serait pas posé ce type de question, elle ne serait pas rentrée dans ce type de problématique. Je pense en effet que si elles peuvent s'intéresser aux mêmes objets d'analyse, AC et $\mathrm{AD}$ ne leur posent les mêmes questions parce qu'elles n'ont pas les mêmes visées.

Un mot, avant de conclure, sur les relations entre images et discours verbal que j'aurais pu développer davantage. En fait, ce n'est pas un problème majeur; dans le couple audio-visuel tel qu'il se réalise au JT, l'audio se limite le plus souvent au verbal (voire au discursif/ domaine largement balisé), dont on n'exclut pas la dimension vocale, plus charnelle.

Quant au " visuel », le domaine est évidemment vaste et variable en fonction des émissions prises en compte, chacune déterminant, en fonction de sa scénographie propre, les éléments pertinents, que ce soit au niveau de l'image externe, avec les impératifs filmiques qui sont les siens (angles de prises de vues, mouvements de caméra, etc.) ou au niveau de l'image interne, du «montré », avec les éléments relevant du kinésique, du proxémique, du figuratif, les deux dimensions de l'image (interne et externe) étant en interaction permanente. Mais ici comme ailleurs, il ne s'agit pas de faire une analyse systématique de ces éléments, un recueil exhaustif de ces données, mais de repérer des moments clés.

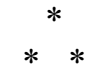

Il ne suffit pas de rappeler l'importance de ces éléments co-textuels et contextuels, il faut les intégrer dans la problématique de recherche, i.e. annuler la différence énoncé/énonciation que j'ai posée plus haut pour expliciter les choses; production/émission/réception doivent être conjointes dans l'analyse. 
Ce principe une fois posé, et une fois affirmé le fait que l'AD me parait préférable pour l'analyse des discours télévisuels parce qu'adéquate à son objet d'étude, il est clair que sa grande faiblesse par rapport à l'AC réside dans la faible reproductibilité de la méthode. Contrairement à l'AC qui se fonde sur des opérations parfaitement routinisées (repérage d'unités lexicales ou syntaxiques, classement, comptage, etc., puis interprétation), l'AD se fonde sur des éléments en partie intuitifs; en dehors des principes précédemment énoncés, chaque ensemble discursif va susciter les éléments de sa propre analyse (on a évoqué les critères d'analyse endogènes en $\mathrm{AD}$ ), chaque problématique va engendrer les outils spécifiques de son exploration.

Tout est chaque fois à réinventer, avec le risque, à chaque fois, de ne rien trouver, contrairement, sans doute, à ce qui se produit en analyse de contenu où une fois que la machine est lancée, elle finit toujours par produire un résultat : il y a toujours des choses à compter (plus ou moins pertinentes, évidemment) et toujours des commentaires à faire à ce propos (plus ou moins pertinents eux aussi). C'est sans doute aussi cette facilité initiale de l'AC qui explique en partie son succès. En $\mathrm{AD}$ en revanche, il existe un réel saut qualitatif entre les principes consistant à intégrer les éléments contextuels et co-textuels propres au discours TV et la pratique de l'analyse.

Il peut arriver qu'AD et AC travaillent sur un même objet, approché de deux façons différentes. Mais le plus souvent me semble-t-il, elles ne privilégient pas les mêmes objets d'analyse, et surtout elles ne leur posent pas les mêmes questions. On a évoqué plus haut le fait que pour travailler sur des séries longues, dans le temps notamment, ou sur des données discontinues, l'AC paraissait moins inadéquate. En quoi $\mathrm{AD}$ et $\mathrm{AC}$ sont, si l'on veut terminer de façon œcuménique, complémentaires... 